\title{
Point of care ultrasound in acute kidney injury - rapid and reliable imaging at the point of admission
}

\author{
Authors: Samiksha Nepal, ${ }^{\mathrm{A}}$ Nicholas Smallwood ${ }^{\mathrm{A}}$ and Martin Dachsel ${ }^{\mathrm{A}}$
}

\section{Introduction}

The aim of this study was to assess the accuracy of renal tract point of care ultrasound (POCUS) according to focused acute medical ultrasound (FAMUS) curriculum to identify and grade hydronephrosis in patients admitted to the acute medical unit (AMU) with acute kidney injury.

\section{Materials and methods}

This was a prospective observational study of a convenience sample of patients older than 18 years admitted to AMU with acute kidney injury. A POCUS was performed by acute physicians according to FAMUS curriculum. A departmental renal ultrasound / computed tomography of the renal tract was then conducted; the radiologist was blind to POCUS result. The objective was to determine the diagnostic performance of POCUS performed by acute physicians for the detection of hydronephrosis using a departmental scan as a gold standard.

\section{Results}

A total of 54 patients were included in the study conducted from July 2018 to December 2018. Out of 54 patients, one patient had a single kidney (left nephrectomy), one patient had three kidneys (transplant) and two patients were found to have atrophic kidneys. A total of 106 kidneys were scanned, with 102 (96.2\%) adequately imaged by POCUS; 96 kidneys were used for comparative analysis; hydronephrosis was noted in five patients on POCUS. Sensitivity of POCUS was $90 \%$ while its specificity was $100 \%$ with a positive predictive value of $100 \%$ and negative predictive value of $99 \%$. The average time to POCUS from initial scan request was 40 minutes and time to departmental scan report from initial scan request was 19 hours 22 minutes. The average time saved by POCUS was 18 hours 20 minutes.

\section{Conclusion}

Our study demonstrates that POCUS as a part of patient management in acute kidney injury is quick, accurate and saves time compared to traditional departmental imaging. It highlights that acute physicians can accurately identify hydronephrosis in patients with acute kidney injury.

\section{Conflicts of interest}

None declared. 